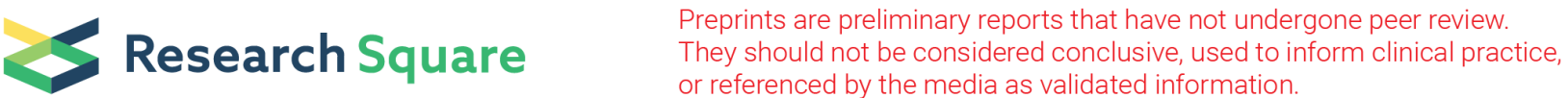 \\ Degree of Satisfaction-based Adaptive Interaction in Spatial Prisoner's Dilemma
}

\author{
Jiaqi Li ( $\sim$ lijiaqi006@126.com ) \\ Hexi University \\ Jianwu Dang \\ Lanzhou Jiaotong University \\ Jianlei Zhang \\ Nankai University \\ Zengqiang Chen \\ Nankai University \\ Matthias Dehmer \\ Technische Universitat Darmstadt
}

\section{Research Article}

Keywords: Evolutionary game, Degree of satisfaction, Adaptive interaction, Cooperation, Prisoner's dilemma, Spatial structure

Posted Date: November 2nd, 2021

DOl: https://doi.org/10.21203/rs.3.rs-273043/v1

License: (c) (i) This work is licensed under a Creative Commons Attribution 4.0 International License. Read Full License

Version of Record: A version of this preprint was published at Nonlinear Dynamics on January 28th, 2022. See the published version at https://doi.org/10.1007/s11071-021-07137-0. 


\title{
Degree of satisfaction-based adaptive interaction in spatial prisoner's dilemma
}

\author{
Jiaqi Li · Jianwu Dang · Jianlei Zhang - Zengqiang Chen · Matthias Dehmer
}

Received: date / Accepted: date

\begin{abstract}
To study why the altruistic cooperation behavior can emerge and maintain among egoistical individuals, researchers across several disciplines have made great contributions for the solutions of this fascinating problem. Ordinarily, the spatial structure is a most-often used framework to investigate the cooperative dynamics of evolutionary game. However, very few researchers take into account the reaction of evolutionary game dynamics to interactive intensity between individuals. On account of this, we propose a computational model of automatic adjustment the interactive intensity based on individual's degree of satisfaction to study the iterated prisoner's dilemma game in a twodimensional square lattice. In this model, selfish individual considers whether the benefits obtained from the other party satisfies its own requirements to determine the intensity of interaction from it to the other party. More specifically, the interactive intensity from an individual $x$ to its some neighbor $y$ is driven by the relations between $x$ obtained current benefit from $y$ (denoted by $P_{x \rightarrow y}$ ) and $x$ 's satisfaction payoff (denoted by $S_{p}$ ). If $P_{x \rightarrow y}>S_{p}, x$ will increase the intensity of interaction from itself to $y$; On the contrary, if $P_{x \rightarrow y}<S_{p}, x$ will weaken the intensity of interaction; Other scenario remain the same. Simulation results show that the proposed mechanism can effectively promote the emergence and maintain of cooperation in population, and the satisfying coefficient $\alpha(0<\alpha<1)$ plays an essential role on

\section{J. Li}

Institute of Intelligent Information, Hexi University, Zhangye 734000, China.E-mail: lijiaqi006@126.com

J. Li and J. Dang

School of Electronic and Information Engineering, Lanzhou Jiaotong University, Lanzhou 730070, China.

J. Zhang and Z. Chen

The College of Artificial Intelligence, Nankai University, Tianjin 300350, China.

M. Dehmer

Technische Universität Darmstadt, Darmstadt 64289, Germany.
\end{abstract}

cooperation. Interestingly, we found that there are some optimal values $\alpha$ can lead to the best promotion of cooperation. However, individual's overclaim $(\alpha>1)$ is not conducive to the effective promotion of cooperation between selfish individuals even for very small temptations to defect. Our results may contribute to the understanding of cooperative dynamics by considering the reaction of evolutionary game dynamics to network.

Keywords Evolutionary game - Degree of satisfaction · Adaptive interaction - Cooperation - Prisoner's dilemma . Spatial structure

\section{Introduction}

Social dilemmas probably emerge if individual actions succeed in damaging the collective interest $[1,2]$. However, cooperative phenomenon is ubiquitously observed in biological systems and animal species, as well as human society [3-6]. How to understand the widespread cooperative behavior between egoistical individuals is still a constant topic of discussion in recent years. Evolutionary game theory provides a strong mathematical tool to study the cooperative phenomena within groups of selfish individuals [7-9].

Noticeably, since the remarkable work on spatial evolutionary games [10,11], spatial structure has been widely applied to research the cooperative behaviors in various models of social dilemma such as prisoner's dilemma game (PDG), snowdrift game (SDG), and public goods game (PGG) [12-16]. Subsequently, some quintessential rules, like kin selection, group selection, direct and indirect reciprocity, have been recognized as effective methods to increase the viability of cooperators in a competitive environment and further improve the evolution of cooperation [17-19].

What needs to be emphasized is that the spatial reciprocity, which was first proposed and investigated by Nowak 
and May, has been considered as a very reasonable population structure to promote the cooperation between egoistical individuals. In this ground-breaking work, all individuals are placed on a two-dimensional square lattice, and each individual only has the game relationship with its nearest neighbor. In this framework, the cooperators obtain a chance to inhibit the invasion of the defectors by forming locally and closely cooperative clusters [10]. Inspired by this work, many scholars began to study the cooperative dynamics on the networks with their own characteristics include the small-world networks and scale-free networks [20, 21]. It is found that this kind of network structure plays an important role in the evolution of cooperation. In addition, a large number of mechanisms of promoting cooperation have been recently proposed by considering the social factor, such as rewards and punishments [22,23], reputation [24], aspiration [25], tolerance [26], teaching activity [27], memory [28-30], emotion [31-33], rationality [34,35], restructuring partnership [36], migration [37] and so forth. Additionally, interdependent networks have been considered to be a more suitable framework for the investigation of networked cooperative dynamics, and seemingly insignificant factors in one network can lead to the extraordinary consequences in another network [38-40]. Intriguingly, since Parrondo conceptualized the idea that two losing games can combine to give a winning game, the study of individual behavior under the framework of the Parrondo's paradox has also been fascinated by more and more scholars [41-44].

Nevertheless, in the most of above spatial game models, scholars often assume that intensity of interaction between individuals is sufficiently strong. In other words, an individual always interacts with its immediate neighbor, regardless of time and space. On account of this, Traulsen et al. investigated the effects of random interaction on cooperation by an invariable intensity of interaction which described by a fixed probability. $[45,46]$. Furthermore, considering the continuous change of interactive intensity driven by evolutionary game dynamics, Li et al. proposed some mechanisms about the reaction of evolutionary game dynamics to network connection weights $[7,47]$, but these mechanisms only consider the interior information of interactive result between individuals. In short, whether the intensity of interaction is fixed or dynamic, all these work have enriched the knowledge of evolutionary game cooperation dynamics. Still, it should be emphasized that the satisfaction payoff between two selfish individuals is not necessarily symmetric. On the other hand, an individual's satisfaction payoff not only dependent on the payoff information of interactive result, but also related to the information of individual's local environment. Motivated by these facts, we investigate the networked cooperative dynamics of automatically adjusting the intensity of interaction based on the individual's degree of satisfaction.
The rest of this paper is organized as follows. In Section 2 , we describe the computational model in detail. The simulation results and analysis are arranged in Section 3 . The last part is the conclusion.

\section{Model}

Before we launch into the model in detail, let us consider the population structure that will execute the games. We use a network to characterize the population structure, each node in the network denotes an individual, and each edge represents the pairwise interaction relationship. Furthermore, we attach two directions to each edge of the network, one is from an individual to its neighbor, and the other is from the neighbor to the individual. The weights in the direction represent the unilateral intensity of interaction each other. Through this formulation, only when each party successfully send the interactive signal to opponent with the unilateral interactive intensity, respectively, can they participate in the game. Obviously, for paired two individuals, the larger the amplitude of the unilateral interactive intensity, the higher the probability of successful interaction. Taking use case view as an example, for the paired individuals $x$ and $y$ in Fig. 1, $W_{x \rightarrow y}$ contains two-fold meanings: 1) $x$ is willing to contact with $y$; 2) it denotes the amplitude of the interactive intensity from $x$ to $y$. Taken together, $x$ voluntarily contact with $y$ with the unilateral intensity of interaction $W_{x \rightarrow y}$. Similarly, the same meanings for $W_{y \rightarrow x}$. When $x$ and $y$ succeed in sending the interactive signal to each other with the interactive intensity $W_{x \rightarrow y}$ and $W_{y \rightarrow x}$, respectively, they can attend the game.

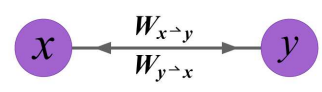

Fig. 1 The pairwise interaction relationship in a network. For the paired individuals $x$ and $y, x$ is willing to contact $y$ with the interactive intensity $W_{x \rightarrow y}$, and $y$ is also voluntarily contact $x$ with the interactive intensity $W_{y \rightarrow x}$. When $x$ and $y$ succeed in sending the interactive signal to each other with the interactive intensity $W_{x \rightarrow y}$ and $W_{y \rightarrow x}$, respectively, they can attend the game.

As usual, we use the symbol $S_{x}(t)$ to indicate the strategy of individual $x$ which changes over time, and the individual $x$ has two simple pure strategies described by the different unit vector to choose.

$S_{x}(t)= \begin{cases}\left(\begin{array}{l}1 \\ 0\end{array}\right) & (\text { when } x \text { adopts } C) \\ \left(\begin{array}{l}0 \\ 1\end{array}\right) & (\text { when } x \text { adopts } D)\end{cases}$

In $E q$. (1), $C$ and $D$ represent the cooperative strategy and the defection strategy, respectively. 
In addition, we choose the PDG as the social dilemma model to study the cooperative dynamics of spatial evolutionary games, because it contains the worst cooperative scenario. Following the usual scheme of PDG [10,39], mutual cooperation enables each party to gain equal benefits $R=1$, but mutual betrayal leads to nothing for both sides $P=0$, if one cooperates and the other defects, the former receives a sucker's payoff $(S=0)$, but its rival obtains a temptation payoff $(T=b>1)$. Thus, the payoff matrix $M$ is as follows

\begin{tabular}{|c|c|c|c|}
\hline & $C$ & $D$ & $C$ \\
\hline & $R$ & $S$ & 1 \\
\hline
\end{tabular}

Hereafter, we begin to present our computational model after the PDG is involved in the directed and weighted square-lattice with periodic boundary conditions. By the way, the square-lattice is made up of $L \times L$ ( $L$ rows, $L$ columns) elements, and each element $e_{L j}(j=1,2, \ldots, L)$ in the $L$ th row is connected to the element $e_{1 j}$ at the corresponding position in the first row, and each element $e_{i L}(i=1,2, \ldots, L)$ in the Lth column is connected to the element $e_{i 1}$ at the corresponding position in the first column. In this way, each element always has four adjacent elements to connect with it.

First, each individual (say $x$ ) performs pairwise interactions with the same strategy and the corresponding intensity of interaction in its all neighbors, and calculates its cumulative return of this round

$P_{x}(t)=\sum_{y \in E_{x}(t)} S_{x}^{T}(t) M S_{y}(t)$,

where $E_{x}(t)$ represents the set of effective neighbors of individual $x$ at time $t$ step. It is noteworthy that individual $x$ probably just successful interaction with its some neighbors during the process of stochastic interaction, in contrast, the interaction with the remaining neighbors are aborted. Under the circumstances, we regard the neighbors who actually take part in game with individual $x$ as its effective neighbors.

Whereafter, the unilateral interactive intensity between the paired individuals is likely to be revised, and the adjusted amplitude will be used as the one of the next round. Specific revision rule is as follows

$W_{x \rightarrow y}(t+1)=W_{x \rightarrow y}(t)+\Delta W_{x \rightarrow y}$,

in $E q .(4), \Delta W_{x \rightarrow y}$ means the variable quantity of the unilateral interactive intensity, and

$\triangle W_{x \rightarrow y}=\xi \tanh (\theta)$.

Namely,

$W_{x \rightarrow y}(t+1)=W_{x \rightarrow y}(t)+\xi \tanh (\theta)$,

here

$\theta=P_{x \rightarrow y}(t)-\alpha \frac{P_{x}(t)}{N_{x}(t)}$.
In order to clearly account for the adaptive adjustment rule [Eq.(6) and Eq.(7)], we define two basic concepts in terms of individual benefits.

Definition 1 (Reference Payoff, $R_{p}$ ) At time $t$ step, we take the average value of the individual's cumulative income at the current round as the reference payoff of individual,

$R_{p}(t)=\frac{P_{x}(t)}{N_{x}(t)}$.

Here, it should be emphasized that $N_{x}(t)$ denotes the number of effective neighbors of the individual $x$ at the current round. Namely, $N_{x}(t)=$ length $\left(E_{x}(t)\right)$.

Definition 2 (Satisfaction Payoff, $S_{p}$ ) Satisfaction payoff, compared with the reference payoff, means the benefit which can satisfy the individual $x$ at the current round,

$S_{p}(t)=\alpha R_{p}(t)$.

Here $\alpha$ denotes the satisfying coefficient, and $\alpha>0$. It characterizes the degree of satisfaction of individual $x$ to the reference payoff $R_{p}(t)$. The bigger the amplitude of $\alpha$, the higher the satisfaction payoff of individual $x$. In other words, the individual's demand for satisfactory payoff increases with the increase of $\alpha$.

For the paired individuals $x$ and $y$, whether individual $x$ adjusts the unidirectional intensity of interaction from itself to its neighbor $y$ depends on the relation between $x$ obtains the payoff from $y$ [denoted by $P_{x \rightarrow y}(t)$ ] and the satisfaction payoff of $x$. When $P_{x \rightarrow y}(t)>S_{p}(t)$, individual $x$ will enhance the value of $W_{x \rightarrow y}(t)$, and the quantity of the enhancement depends on the difference between $P_{x \rightarrow y}(t)$ and $S_{p}(t)$. The higher the difference, the higher the quantity of enhancement. Oppositely, $x$ will weaken that in case of $P_{x \rightarrow y}(t)<$ $S_{p}(t)$, and the quantity of decrease according to the corresponding rule.

In light of the context, let's further explain the origin of Eq.(5). We first set up a method that can roughly calculate variable quantity of interactive intensity

$\triangle W_{x \rightarrow y}^{*}=\operatorname{sgn}(\theta) f(|\theta|)$,

here

$\operatorname{sgn}(\theta)=\left\{\begin{array}{cc}1 & (\theta>0) \\ 0 & (\theta=0) . \\ -1 & (\theta<0)\end{array}\right.$

The combination of Eq.(10) and Eq.(11), we can get

$\Delta W_{x \rightarrow y}^{*}=\left\{\begin{array}{cc}f(\theta) & (\theta>0) \\ 0 & (\theta=0) \\ -f(-\theta) & (\theta<0)\end{array}\right.$

From the Eq.(12), we can see $\Delta W_{x \rightarrow y}^{*}$ is an odd function of $\theta$. On the other hand, $\Delta W_{x \rightarrow y}^{*}$ should be a nonlinear curve of continuous changing inspired by the changing characteristics of relationships in real situation. For these reasons, we use the hyperbolic tangent functions to simulate the changing of $\Delta W_{x \rightarrow y}^{*}$. Namely,

$\Delta W_{x \rightarrow y}^{*}=\tanh (\theta)$. 
Even so, the relationship, very likely, is also a physical quantity of slow change. Owing to $\tanh (\theta) \in(-1,1)$, so we draw a more accurate expression for the variable quantity of interactive intensity $\Delta W_{x \rightarrow y}=\xi \tanh (\theta)$. The parameter $\xi$, the coefficient of the $\tanh (\theta)$, denotes the changing speed of variable quantity of interactive intensity. When $\xi=0$, our computational model is simplified to the traditional model, in which the interactive relationships are immutable decided by initialization. When $\xi>0$, the higher the value of $\xi$, the faster the changing speed.

So far, the revision rule for unidirectional interaction strength can be written as

$W_{x \rightarrow y}(t+1)=W_{x \rightarrow y}(t)+\xi \tanh \left[P_{x \rightarrow y}(t)-\alpha \frac{P_{x}(t)}{N_{x}(t)}\right]$.

Similarly, the neighbor $y$ is also possible to adjust the relationship from itself to $x$, that is $W_{y \rightarrow x}(t+1)=W_{y \rightarrow x}(t)+$ $\xi \tanh \left[P_{y \rightarrow x}(t)-\alpha \frac{P_{y}(t)}{N_{y}(t)}\right]$. These revised intensity of interactions will be used as the interactive strength for the next round game. Additionally, only when $x$ and $y$ succeed in sending the interactive signal to each other with the interactive intensity $W_{x \rightarrow y}(t+1)$ and $W_{y \rightarrow x}(t+1)$, respectively, can they participate in the game at the $t+1$ round.

Finally, individual $x$ will learn from its neighbors so as to make a good profit for the next round. Specifically, individual $x$ randomly selects one of its neighbors (say $y$ ) and adopts $y$ 's strategy with a probability depending on the difference of accumulated payoff at the current round [48]

$z\left[P_{x}(t)-P_{y}(t)\right]=\frac{1}{1+\mathrm{e}^{\left[P_{x}(t)-P_{y}(t)\right] / K}}$.

Here $K$ denotes the noise strength, and $K>0$. When $K \rightarrow 0$ means the deterministic imitation, whereas $K \rightarrow+\infty$ leads to random drift. In this paper, we mainly investigate how the satisfying coefficient $\alpha$ influences the evolution of cooperation in spatial PDG.

\section{Results}

Simulations are implemented on the $L \times L$ directed and weighted lattice. Originally, C and D were uniformly and randomly distributed throughout the population, and the value of the unilateral interactive intensity is randomly distributed between 0 and 1 . It should be pointed out that the dynamical changing of the interactive intensity is likely to lead to the unreasonable phenomenon (the value of interactive intensity exceeds 1 , or is no more than 0 ) during the evolution process of system. So we set the minimum amplitude to 0.0001 and the maximum amplitude to 1 . The most important quantity for characterizing the cooperative behavior of system is the cooperation level, $\rho_{c}$, which is defined as the fraction of cooperators at the stationary state of system. When the sys- tem enters a stable state, we achieve the amplitude of $\rho_{c}$ by averaging the last $m$ rounds of the whole $T_{0}$ rounds

$\rho_{c}=\frac{1}{m} \sum_{t=T_{0}-m}^{T_{0}} \frac{N_{c}(t)}{L \times L}$.

Where $N_{c}(t)$ denotes the number of cooperators at the $t$ round. In the numerical simulation, we set $m=3 \times 10^{3}$ and $T_{0}=10^{4}$ (sometimes $T_{0}$ is even set to $2 \times 10^{4}$.) In addition, in order to get a more accurate value of the cooperation level, each data point reported below is averaged over 100 independent experiments.

In keeping with traditional research method, we first investigate the cooperation level $\rho_{c}$ at the steady state of system as a function of the temptation to betray $b$ for different values of the satisfying coefficient $\alpha$ (see Fig.2).

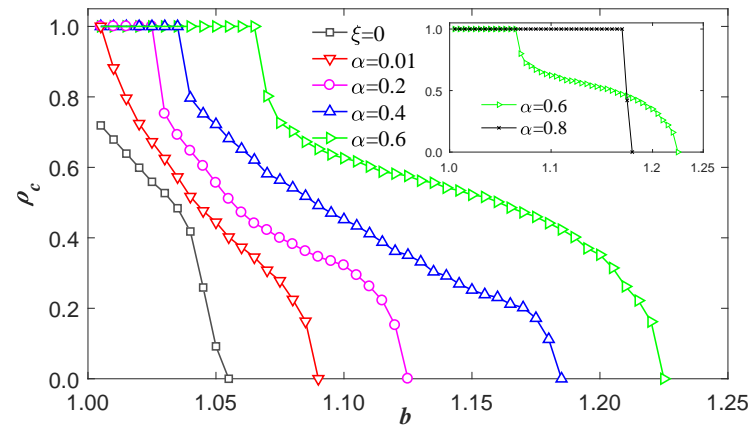

Fig. 2 The cooperation level $\rho_{c}$ at the steady state of system as a function of the temptation to betray $b$ for different values of the satisfying coefficient $\alpha$. The PDG is carried out on a directed and weighted square lattice. For the fixed $\xi=0.1$, compared to the conventional mode $(\xi=0)$, the cooperation level is significantly improved and cooperators sometimes can achieve the completely dominant situation when considering the adaptive interaction based on the degree of satisfaction $(\alpha>0)$. On the other hand, the threshold of cooperative annihilation gradually extends with the gradual increase of $\alpha$. Other corresponding parameters: $L=100, \xi=0.1, K=0.1$.

One can see that, compared to the conventional mode $(\xi=0$, in which the probability of interaction is changeless between paired individuals) of the spatial PDG, $\rho_{c}$ is significantly improved and cooperators sometimes can achieve the completely dominant situation when considering the adaptive interaction based on the degree of satisfaction $(\alpha>0)$. For example, when $\alpha$ is set to a very small amplitude (such as $\alpha=0.01), \rho_{c}$ will rise to a higher amplitude. Furthermore, the threshold at which cooperation disappears is extended to 1.09 (for $\alpha=0.01$ ), whereas that is just close to 1.055 in the traditional case. Whereafter, the threshold of cooperative annihilation gradually extends with the gradual increase of $\alpha$.

However, what surprises us is that the avalanche of cooperation level will appear when $\alpha$ increases to close to 0.8 (see the illustration in Fig.2). Therefore, in order to precisely 
quantify the role of adaptive interaction based on the degree of satisfaction in contributing cooperation, we then investigate the relationship between the cooperation level $\rho_{c}$ at the steady state and the satisfying coefficient $\alpha$ for different values of temptation to betray $b$ (see Fig.3).

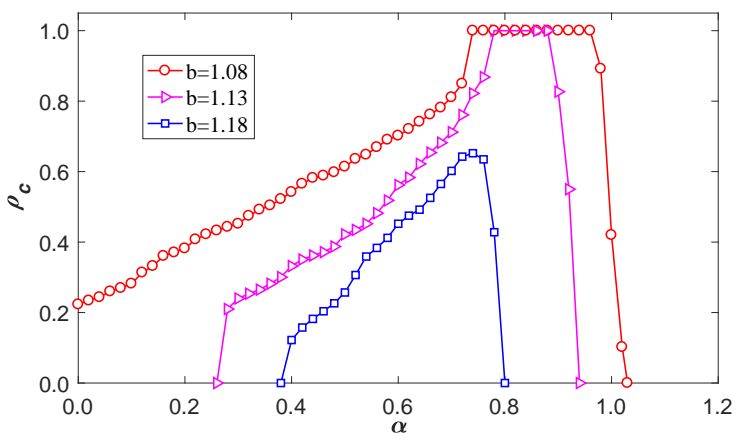

Fig. 3 The relationship between the cooperation level $\rho_{c}$ at the steady state and the satisfying coefficient $\alpha$ for different values of temptation to betray $b$. As you can see, there are some optimal values of $\alpha$, resulting in a plateau of the highest level on cooperation for each small (such as $b=1.08$ ) or moderate (such as $b=1.13$ ) value of $b$. Furthermore, the length of this type of plateau gradually shortens when the value of $b$ is enhanced little by little. Although the plateau of high cooperation has disappeared when $b$ increases to the large value (such as $b=1.18$ ), there still exists an optimal value $\alpha$, resulting in the most conducive to cooperation. Other corresponding parameters: $L=100, \xi=0.1$, $K=0.1$.

As you can see, there are some optimal values $\alpha$, resulting in a plateau of the highest level on cooperation for each small value of $b$ (such as $b=1.08$ ). Furthermore, the length of this type of plateau gradually shortens when the value of $b$ is enhanced little by little (e.g., from $b=1.08$ to $b=1.13$ ). But for a large value of $b$ (such as $b=1.18$ ), this type of plateau will disappear, in spite of what happened, there still exists an optimal value $\alpha$, resulting in the most conducive to cooperation.

Unexpectedly, when $\alpha>1$, it is not the best way to promote cooperation in the population regardless of the value of $b$. In other words, individual's overclaim (only when an individual obtained the payoff from its neighbor exceeds the reference payoff, will the individual enhance the interactive intensity from it to the neighbor; otherwise it will weaken.) is not conducive to the effective promotion of cooperation between selfish individuals. Moreover, the higher the amplitude of $\alpha(\alpha>1)$, the worse the effect, it is true even for those smaller $b$ (see Fig.4). Combined with Fig.3 and Fig.4, we can see that regardless of whether $b$ is a very small amplitude (such as $b=1.01$ ) or a very large amplitude (such as $b=1.18$ ), there are some optimal values $\alpha$ that can effectively promote the cooperation in the population.

From here we can get a conclusion according to the range of the temptation to defect $b$, that is, the satisfying coefficient $\alpha^{\text {opt }}$ that can most effectively promote cooperation in

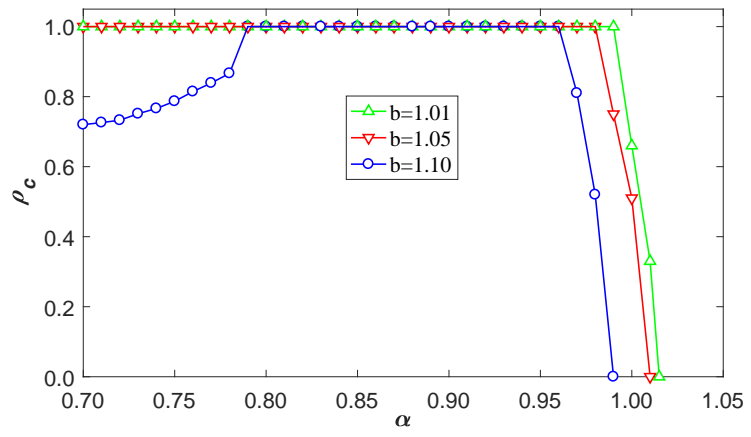

Fig. 4 The relationship between the cooperation level $\rho_{c}$ at the steady state and the satisfying coefficient $\alpha$ for some smaller values of temptation to betray $b$. It is observed that individual's overclaim is not conducive to the effective promotion of cooperation between selfish individuals even for some very small $b$. Moreover, the higher the amplitude of $\alpha(\alpha>1)$, the worse the effect of promoting cooperation. Other corresponding parameters: $L=100, \xi=0.1, K=0.1$.

the population satisfies

$$
\left\{\begin{array}{c}
\left\{\alpha^{o p t} \mid \alpha_{\min } \leq \alpha^{o p t} \leq \alpha_{\max }\right\}=\arg \max _{\alpha \in(0,1)} \rho_{c}(1<b<1.175) \\
\alpha^{o p t}=\arg \max _{\alpha \in(0,1)} \rho_{c} \quad(1.175 \leq b<1.225)
\end{array}\right.
$$

On balance, these results show that the adaptive interaction between paired individuals, by considering the degree of satisfaction, can greatly promote cooperation in spatial prisoner's dilemma.

Whereafter, we collect some typical snapshots about the microscopic evolution of individual behavior in population to enhance our understanding for the effect of adaptive interaction based on the degree of satisfaction. Here, we choose a moderate amplitude of $b$ (such as $b=1.13$ ) and an appropriate value of $\alpha$ (such as $\alpha=0.7$ ) to investigate the dynamic behaviors of individual, as shown in Fig.5.

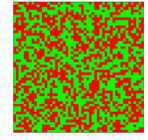

(a)

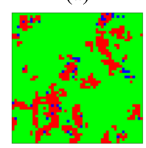

(f)

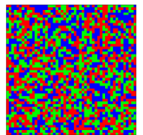

(b)

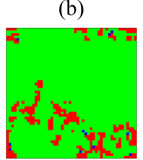

(g)

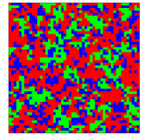

(c)

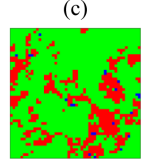

(h)

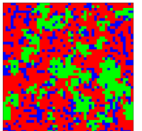

(d)

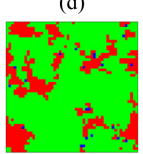

(i)

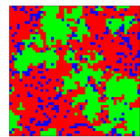

(e)

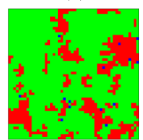

(j)
Fig. 5 The evolution of individuals with different roles (red, green and blue represent defectors, cooperators and isolates, respectively.) on a square lattice for $b=1.13$ and $\alpha=0.7$ at several representative time steps. Specifically, (a) $t=0\left[\rho_{c}(0)=0.4957\right]$; (b) $t=1$ $\left[\rho_{c}(1)=0.3271\right]$; (c) $t=20\left[\rho_{c}(20)=0.2515\right]$; (d) $t=50\left[\rho_{c}(50)=0.3102\right]$; (e) $t=100 \quad\left[\rho_{c}(100)=0.3842\right] ; \quad$ (f) $t=300 \quad\left[\rho_{c}(300)=0.7226\right] ; \quad(\mathrm{g})$ $t=600\left[\rho_{c}(600)=0.8867\right]$; (h) $t=1000\left[\rho_{c}(1000)=0.6588\right]$; (i) $t=3000$ $\left[\rho_{c}(3000)=0.6750\right]$; (j) $t=5000\left[\rho_{c}(5000)=0.6770\right]$. Other corresponding parameters: $L=100, \xi=0.1, K=0.1$. 
From the perspective of the cooperators' experience, the entire evolution of the system can be broken down into three phases: enduring (END) phase, expanding (EXP) phase, and equilibrium (EQU) phase. Originally, the strategy adopted by each individual is initialized to $\mathrm{C}$ or $\mathrm{D}$ with the probability 0.5 , so all individuals are evenly distributed in the whole network with two different states [Fig.5(a)]. Nevertheless, once the system is activated, nearly a third of the individuals are likely to lose the opportunity of getting in touch with their all partners, and they evenly exist in the network as the status of isolates because of the very weak paired relationship assigned by the initialization [Fig.5(b)]. During the END stage, a large number of individuals are more likely to choose betrayal strategies regardless of the friendship to satisfy selfish desires in the social dilemma system, thus the defectors clusters (D-clusters for short) will naturally form by clustering. Unfortunately, a few of defectors located at D-clusters are isolated due to the weaker and weaker interactions. But for all this, there are still some committed cooperators who successfully survive in the final of END phase by forming cooperation clusters (C-clusters for short) [Fig.5(c)]. What should be stressed was the size of C-clusters, although they are very small at the end of END phase, the interactive relation is very close between cooperators because an individual probably is satisfied with the benefit derived from its cooperative neighbor, given that exists the defective parters around it, which leads to the increase of interactive intensity between them, and the higher the frequency of interaction, the closer the contact. Meanwhile, an opposite dynamic scenario is taking place in each D-cluster. In other words, as time goes by, the interactive relation is getting worse and worse between defectors as long as the defector has cooperative neighbors who located at the boundary of D-cluster. Naturally, the incompact D-clusters are apt to be destroyed by close C-clusters, and those captives are easy to be turned into cooperators by learning. In addition, the cooperators unceasingly fulfill the duty of rescuing isolates. As a consequence, the scale of C-clusters are constantly enlarged over time [from Fig.5(d) to Fig.5(g)]. To our great surprise, the scale of C-clusters are not always growing during the entire EXP phase. Specifically, at time $t=600$ step, cooperators show doughty control ability in population and generate the large-scale connected components [Fig.5(g)]. Unfortunately, the advantage of cooperators only can last for a period of time. Shortly afterwards the defectors redouble their efforts and blow the clarion of counterattack to cooperators, which leads to the shrink of C-clusters in size but the corresponding swell for D-clusters [Fig.5(h)]. At last, after the stinging confrontations between cooperators and betrayers, the system goes into a relatively stable state [Fig.5(i) and Fig.5(j)].

To more clearly explain the evolution process of these snapshots, we further investigate the driving force of dynamic evolution of individual behavior. Hereon, we use the method by combining the average payoff (abbreviated as AP) of individual located at the boundary and the cooperation level to study the essential causes of the changing of individual behavior (see Fig.6).

Seen from the panel (a) in Fig.6, the AP of defectors almost always is higher than that of cooperators during END phase, this means the probability $\left(z\left[P_{C}(t)-P_{D}(t)\right]\right)$ that $\mathrm{C}$ learns from $\mathrm{D}$ is higher than the probability $\left(z\left[P_{D}(t)-P_{C}(t)\right]\right)$ that D learns from $\mathrm{C}$ according the Eq.(14). Therefore, the cooperators gradually disappear during this stage, and the cooperation level declines accordingly [see the panel (b) in Fig.6). However, the situation has changed since entering the EXP phase, the AP of cooperators not only catches up with the one of opponents, but surpasses it. Besides, the difference between the values of their AP is becoming bigger and bigger over time. As a result, the expansion speed of C-clusters is slow at the beginning of the EXP period, but then it is getting faster and faster, and this series of dynamic changes in C-clusters directly determine the trajectory of the cooperation level. It should be noted that the AP of defectors also keeps going up and up in the entire EXP phase, but that of cooperators starts to descend at the end of EXP phase, which lead to the transient equilibrium at approximately $t=600$, at this point, the cooperation level arrives at its peak. In quick succession, the AP of defectors going beyond that of cooperators again, which means the defectors restart a counteroffensive to cooperators. We can see clearly that although this counterattack of defectors do not achieve the ultimate great victory, it at least weaken the advantage of the C-cluster. After the further advancement of the system evolution, a balanced competition between cooperators and defectors ultimately emerges in population (see the EQU phase).

As a matter of fact, after interactions between two cooperators, either party could be satisfied with the mutually beneficial outcome because the benefit obtained from the other possibly is higher than their respective satisfaction payoff when $0<\alpha<1$. Without a doubt, they will heighten the awareness of interaction in the next round, which further increases the chances of profit. The more the opportunities of interaction, the more the times of satisfactory situation. It shows that there may be a positive feedback effect between the degree of satisfaction and the interactive intensity. In contrast, for the interaction between defectors, the negative feedback effect, in all probability, is formed between the degree of satisfaction and the interactive intensity. Because of this, the D-clusters are liable to demolished by cooperators, and those starveling betrayers are easy to be turned into cooperators by learning.

In order to deeply understand the positive feedback effect in the system, we plot some typical snapshots of system 




Fig. 6 Respective average payoffs of cooperators and defectors located at the boundary, and the corresponding cooperation level in population. Even though the average payoff of cooperators is lower than that of defectors during the END stage, soon after, the average payoff of cooperators not only succeeds in pursuing that of defectors, and surpasses it in the EXP stage. But as the system further evolves, it begins to decline until they almost have the same amplitudes. Spontaneously, the cooperation level correspondingly follows the trajectory from initial decline to gradual rise and then to stable state. Other corresponding parameters: $L=100, \xi=0.1, K=0.1$.

at steady state for the fixed $b=1.13$ and the different values of $\alpha$, as shown in Fig.7.

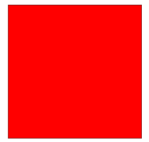

(a)

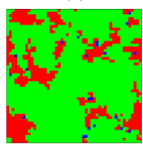

(d)

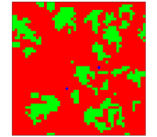

(b)

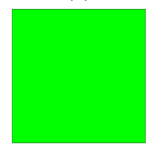

(e)

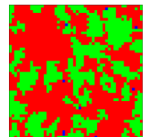

(c)

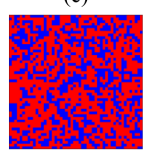

(f)
Fig. 7 Some typical snapshots of system (red, green and blue represent defectors, cooperators and isolates, respectively.) on a square lattice for $b=1.13$ and the different values of $\alpha$ at steady state. Specifically, (a) $\alpha=0.1\left[\rho_{c}(\alpha)=0\right]$; (b) $\alpha=0.3\left[\rho_{c}(\alpha)=0.2815\right]$; (c) $\alpha=0.5$ [ $\left.\rho_{c}(\alpha)=0.4325\right]$; (d) $\alpha=0.7\left[\rho_{c}(\alpha)=0.6588\right]$; (e) $\alpha=0.9\left[\rho_{c}(\alpha)=1\right]$; (f) $\alpha=1\left[\rho_{c}(\alpha)=0\right]$. Other corresponding parameters: $L=100, \xi=0.1$, $K=0.1$.

In Fig.7(a), we can see that the cooperators are thoroughly extinguished when $b=1.13$ and $\alpha=0.1$. Just imagine, under this circumstances, there is still the extremely faint positive feedback effect in the system, and some speckled C-clusters can also be formed gradually by the positive feedback effect during the END stage, but the resistibility of cooperators located at the boundary is too unsubstantial, so those C-clusters are easily demolished and all cooperators are wiped out by traitors. As the growth of $\alpha$ [from
Fig.7(b) to Fig.7(e)], the positive feedback effect is becoming more and more conspicuous, the resistibility of cooperators located at the boundary are also becoming more and more stronger, even their roles maybe undergo the qualitative change (from defense to attack). Therefore, the size of C-clusters increases with increasing $\alpha(\alpha<1)$. Nevertheless, when $\alpha$ close to even exceed 1, the positive feedback effect almost disappear even the positive feedback loop was replaced by the negative feedback loop, because in this situation, the individual may also be dissatisfied with the neighbor within the $\mathrm{C}$-clusters. Thus the weak $\mathrm{C}$-clusters, which are formed by spatial reciprocity, will collapse without being attacked. It should be pointed out that the negative feedback effect also disappears when the cooperators are completely extinct. At this moment, the defectors and the isolators will coexist in the population [Fig.7(f)].

Hereto, we want to mention the payoff matrix $M$, which is adopted by us, is a weak PDG. For all this, it is still widely used by scholars because it does not change some qualitative results $[10,49]$. Remarkably, some scholars have proposed an extended payoff matrix about social dilemmas [50]

$$
\begin{aligned}
& \begin{array}{llll}
C & D & C & D
\end{array} \\
& M_{E}={ }_{D}^{C}\left(\begin{array}{ll}
R & S \\
T & P
\end{array}\right)={ }_{D}^{C}\left(\begin{array}{cc}
1 & -D_{r} \\
1+D_{g} & 0
\end{array}\right) .
\end{aligned}
$$

When $D_{g}>0$ and $D_{r}>0$, the game is a PDG. Thus, we further study the model of adaptive interaction based on the degree of satisfaction in the extended PDG by adjusting the parameters $\left(D_{g}>0\right.$ and $\left.D_{r}>0\right)$ of dilemma strength (see 

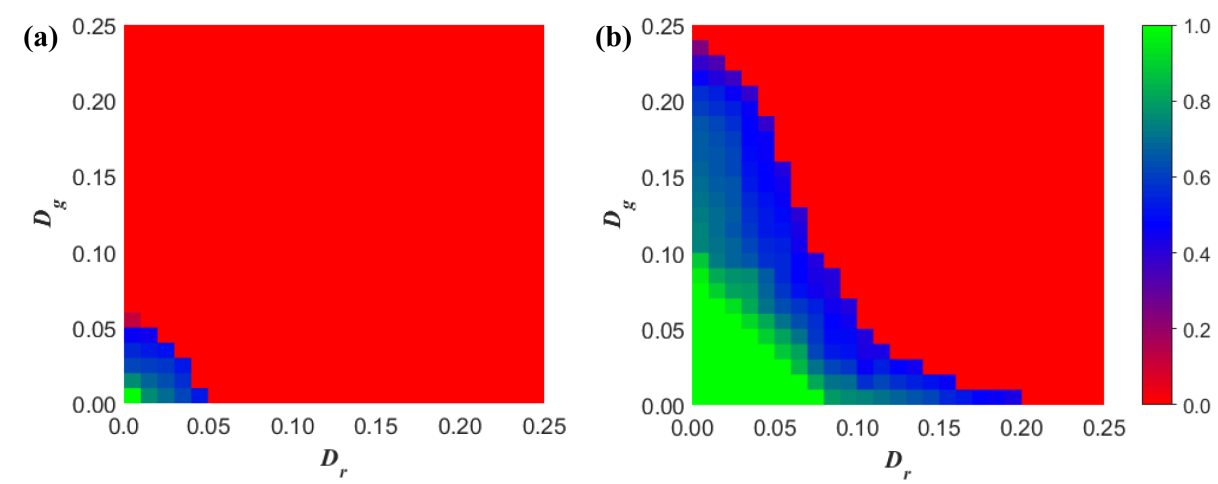

Fig. 8 The cooperation level in population by using the extended payoff matrix. The cooperation level of adaptive interaction based on the degree of satisfaction, compared to the conventional interaction mode $(\xi=0)$, is still greatly increased. Left panel: Conventional interaction mode; Right panel: The adaptive interaction by considering the degree of satisfaction for the fixed $\alpha=0.7$. Other corresponding parameters: $L=100, \xi=0.1$, $K=0.1$.
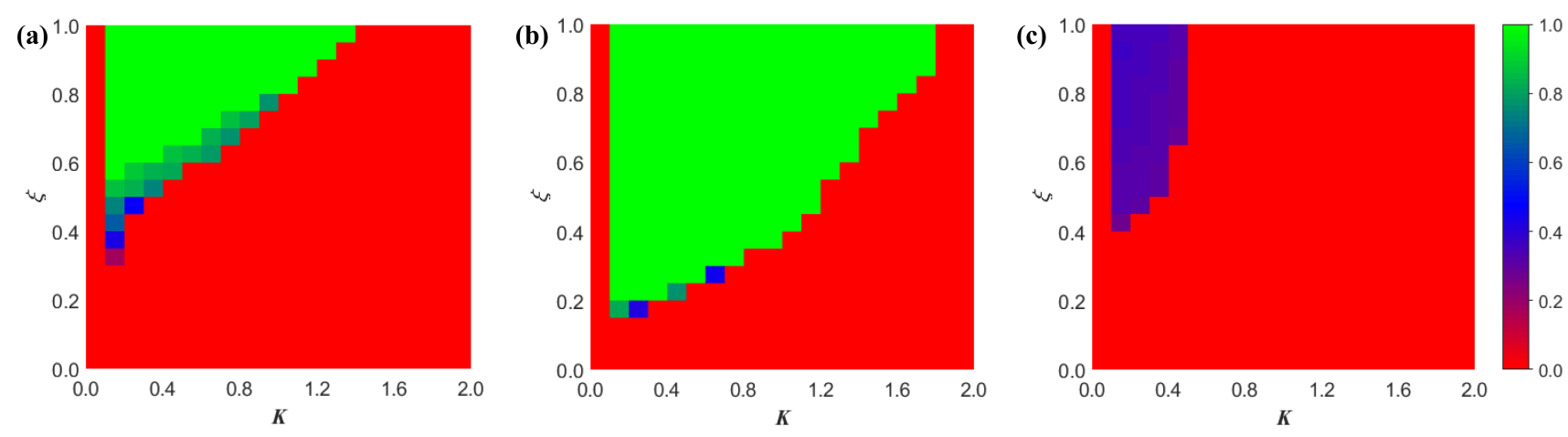

Fig. 9 The cooperation level $\rho_{c}$ as a function of noise strength $K$ and the coefficient of the variable quantity of interactive intensity $\xi$. The qualitative result, there are some optimal values $\alpha$ can warrant the best environment for spreading of cooperation, is not changed. (a) panel: $\alpha=0.1 ;$ (b) panel: $\alpha=0.5$; (c) panel: $\alpha=1$. Other corresponding parameters: $L=100, D_{g}=0.3, D_{r}=0.1$.

Fig.8). Satisfactorily, when consider the more severe dilemma strength (decided by $D_{g}$ and $D_{r}$ ), the cooperation level of adaptive interaction based on the degree of satisfaction in spatial PDG, compared to the conventional interaction mode $(\xi=0)$, is still greatly increased.

It should be noted that the noise strength $K$ and the coefficient of the variable quantity for interactive intensity $\xi$ are directly set as 0.1 by us in the above numerical simulation experiments, we have not taken into account the effect of change of these parameters on the cooperation level. Therefore, we further investigate the cooperation level $\rho_{c}$ as a function of $K$ and $\xi$, as shown in Fig.9.

On the whole, there are some optimal values $\alpha$ (such as $\alpha=0.5$ ) can warrant the best environment for spreading of cooperation with the change of $K$ and $\xi$ (see Fig.9). However, the satisfaction level of individual too low (such as $\alpha=0.1$ ) or too high (such as $\alpha=1.0$ ) is not conducive to cooperation, especially the latter case. Furthermore, it is favorable for cooperation when the noise intensity $K$ is around 0.1 . Specially, the cooperation phenomenon in the population will disappear when $K \rightarrow 0$, regardless of the value of $\xi$. This phenomenon shows that appropriate noise can effectively promote the spread of cooperation. By the way, in order to facilitate cooperation propagation in a high-noise environment, the amplitude of $\xi$ should be increased as compensation. In other words, cooperation can emerge only if $\xi$ increase with the increase of $K$. The reason is that the ability of individuals in learning cooperation behavior will be reduced with the increase of noise in our model, which further depresses the positive feedback effect between the degree of satisfaction and the interactive intensity. Fortunately, this positive feedback effect can be compensated even enhanced by increasing the rate of change for interaction intensity.

Finally, what we would like to indicate here is that the results reported in this paper are robust with regard to the network size (including the small-scale network such as $50 \times 50$ and the large-scale network such as $200 \times 200$ ). Besides, when the asynchronous updating fashion of individual strategy is introduced into our model, we find the qualitative result$\mathrm{s}$ remains almost the same. In the future, we will consider the cooperative dynamics on multi-layer networks and the related applications such as the transmission of virus [51, 52] based on our mechanism. Furthermore, inspired by the works under the framework of the Parrondo's paradox [4144], we will further investigate the adaptive interaction of whether a combination of two losing games can lead to a 
winning outcome under severe social dilemma, work along this line is in progress.

\section{Conclusion}

In real systems, the intensity of interactions between individuals should be driven by a variety of factors. Here, we propose a computational model of automatic adjustment the interactive intensity, by considering the degree of satisfaction, to study the iterated prisoner's dilemma game in a twodimensional square lattice. In this model, when an individual is satisfied with the benefit obtained from the other party, it will increase the interactive intensity from itself to the neighbor; Conversely, it will weaken that if is not satisfied. Furthermore, the variable quantity of interactive intensity depends on the difference between the satisfaction payoff and the benefit obtained from the other party. The results show that the proposed mechanism can effectively promote the emergence and maintain of cooperation in population, and the satisfying coefficient $\alpha(0<\alpha<1)$ plays an essential role on cooperation. For a fixed temptation value, regardless of the value is small or moderate, there are some optimal values $\alpha$, resulting in a plateau of the highest level on cooperation. Moreover, for a large temptation value, there still exists an optimal value $\alpha$, resulting in the most conducive to cooperation, although the plateau of high cooperation has disappeared. However, individual's overclaim $(\alpha>1)$ is not conducive to the effective promotion of cooperation between selfish individuals regardless of the value of temptation. By analysis, we can find out the essential reason of for promoting cooperation is mainly attributed to the positive feedback effect between the degree of satisfaction and the interactive intensity. Maybe for this reason, rather than just relying on the spatial reciprocity, the cooperative behavior can be effectively transmitted in population.

\section{Acknowledgements}

This research is supported by National Natural Science Foundation of China (No.61963013).

\section{Compliance with ethical standards}

Conflict of interest:The authors declare that they have no competing interests.

\section{References}

1. EL-Seidy, E.:The effect of noise and average relatedness between players in iterated games. Appl. Math. Comput. 269, 343-350 (2015)
2. Zhang, J.L., Chen, Z.Q., Liu, Z.X.:Fostering cooperation of selfish agents through public goods in relation to the loners. Phys. Rev. E 93(3), 032320 (2016)

3. Axelrod, R.:The Evolution of Cooperation. Basic Books, (2006)

4. Tanimoto, J.:Fundamentals of Evolutionary Game Theory and its Applications. Springer, (2015)

5. Luo, C., Zhang, X.L., Liu, H., Shao, R.:Cooperation in memorybased prisoner's dilemma game on interdependent networks. Physica A 450, 560-569 (2016)

6. Xiao, Y.P., Chen, D.Q., Wei, S.H., Li, Q., Wang, H.H., Xu, M.: Rumor propagation dynamic model based on evolutionary game and anti-rumor. Nonlinear Dyn. 95(1), 523-539 (2019)

7. Li, J.Q., Zhang, C.Y., Sun, Q.L., Chen, Z.Q., Zhang, J.L.:Changing the Intensity of Interaction Based on Individual Behavior in the Iterated Prisoner's Dilemma Game. IEEE Trans. Evol. Comput. 21(4), 506-517 (2017)

8. Zhang, J.L., Xu, Z.M., Liu, Z.X., Chen, Z.Q.: Effects of strategy switching and network topology on decision-making in multiagent systems. Int. J. Syst. Sci 49(9), 1934-1949 (2018)

9. Tanimoto, J.:Evolutionary Games with Sociophysics: Analysis of Traffic Flow and Epidemics. Springer, (2019)

10. Nowak, M.A., May, R.M.:Evolutionary games and spatial chaos. Nature 359(6398), 826-829 (1992)

11. Ariful Kabir, K.M., Tanimoto, J., Wang, Z.:Influence of bolstering network reciprocity in the evolutionary spatial Prisoner's Dilemma game: a perspective. Eur. Phys. J B 91, 312 (2018)

12. Szabó, G., Fath, G.:Evolutionary games on graphs, Phys. Rep. 446(4), 97-216 (2007)

13. Zhang, J.L., Zhang, C.Y., Cao, M.:How insurance affects altruistic provision in threshold public goods games. Sci Rep 5, 9098 (2015)

14. Tanimoto, J.:Simultaneously selecting appropriate partners for gaming and strategy adaptation to enhance network reciprocity in the prisoner's dilemma. Phys. Rev. E 89(1), 012106 (2014)

15. Santos, F.C., Pacheco, J.M.: Evolutionary dynamics of social dilemmas in structured heterogeneous populations. Proc Natl Acad Sci USA 103(9), 3490-3494 (2006)

16. Liu, L.J., Chen, X.J., Perc, M.:Evolutionary dynamics of cooperation in the public goods game with pool exclusion strategies. Nonlinear Dyn. 97(1), 749-766 (2019)

17. Nowak, M.A.:Five rules for the evolution of cooperation. Science 314(5805), 1560-1563 (2006)

18. Nowak, M.A., Sigmund, K.:Evolution of indirect reciprocity. Nature 437(7063), 1291 (2005)

19. Ito, H., Tanimoto, J.:Scaling the phase-planes of social dilemma strengths shows game-class changes in the five rules governing the evolution of cooperation. Roy. Soc. Open Sci. 5(10), 181085 (2018)

20. Iotti, B., Antonioni, A., Bullock, S., Darabos, C., Tomassini, M., Giacobini, M.:Infection dynamics on spatial small-world network models. Phys. Rev. E 96(5), 052316 (2017)

21. Ichinose, G., Sayama, H.:Invasion of cooperation in scale-free networks: accumulated versus average payoffs. Artif. Life 23(1), 2533 (2017)

22. Wu, Y., Zhang, B., Zhang, S.H.:Probabilistic reward or punishment promotes cooperation in evolutionary games. Chaos, Soliton Fract 103, 289-293 (2017)

23. Liu, J.Z., Meng, H.R., Wang, W., Li, T., Yu, Y.:Synergy punishment promotes cooperation in spatial public good game. Chaos, Soliton Fract 109, 214-218 (2018)

24. Cuesta, J.A., Gracialázaro, C., Ferrer, A., Moreno, Y., Sánchez, A.:Reputation drives cooperative behaviour and network formation in human groups. Sci. Rep. 5, 7843 (2015)

25. Shen, C., Chu, C., Shi, L., Perc, M., Wang, Z.:Aspiration-based coevolution of link weight promotes cooperation in the spatial prisoner's dilemma game. Roy. Soc. Open Sci. 5(5), 2054-5703 (2018) 
26. Chen, X.J., Wang, L.:Cooperation enhanced by moderate tolerance ranges in myopically selective interactions. Phys. Rev. E 80(4), 046109 (2009)

27. Szolnoki, A., Perc, M.:Coevolution of teaching activity promotes cooperation. New J. Phys. 10, 043036 (2008)

28. Ye, W., Feng, W., Lü, C., Fan, S.:Memory-based prisoner's dilemma game with conditional selection on networks. Appl. Math. Comput. 307, 31-37 (2017)

29. Gao, L., Wang, W., Shu, P.P., Gao, H., Braunstein, L.A.:Promoting information spreading by using contact memory. EPL 118, 18001 (2017)

30. Li, J.Q., Dang, J.W., Zhang, J.L.:Length of information-based bidirectional choice in spatial prisoner's dilemma. Appl. Math. Comput. 369, 124837 (2020)

31. Dai, Y.Y., Zhan, G.J., Ye, Y., Bao. W., Wen, T., Cheong, K.H., Xie, N.G.:Game dynamics of emotion evolution based on the Moran process. Chaos 31, 033153 (2021)

32. Wang, L., Ye, S.Q., Cheong, K.H., Bao, W., Xie, N.G.:The role of emotions in spatial prisoner's dilemma game with voluntary participation. Physica A 490, 1396-1407 (2018)

33. Xie, N.G., Zhen, K.X., Wang, C., Ye, Y., Wang, L.:Evolution of cooperation driven by the diversity of emotions. Connect. Sci. 27(1), 89-101 (2015)

34. Chen, Y.Z., Huang, Z.G., Wang, S.J., Zhang, Y., Wang, Y.H.:Diversity of rationality affects the evolution of cooperation. Phys. Rev. E 79(5), 055101 (2009)

35. Ye, W.X., Fan, S.H.: Evolutionary snowdrift game with rational selection based on radical evaluation. Appl. Math. Comput. 294, 310-317 (2017)

36. Li, J.Q., Zhang, C.Y., Sun, Q.L., Chen, Z.Q.:Coevolution between strategy and social networks structure promotes cooperation. Chaos, Soliton Fract 77, 253-263 (2015)

37. Li, Y., Ye, H., Zhang, H.:Evolution of cooperation driven by social-welfare-based migration. Physica A 445, 48-56 (2016)

38. Wang, Z., Wang, L., Szolnoki, A., Perc, M.:Evolutionary games on multilayer networks: a colloquium. Eur. Phys. J. B 88(5), 124 (2015)

39. Shi, L., Shen, C., Geng, Y.n., Chu, C., Meng, H.R.:Winnerweaken-loser-strengthen rule leads to optimally cooperative interdependent networks. Nonlinear Dyn. 96(1) 49-56 (2019)

40. Luo, C., Zhang, X.L., Zheng, Y.J.:Chaotic evolution of prisoner's dilemma game with volunteering on interdependent networks. Commun. Nonlinear Sci. 47, 407-415 (2017)

41. Tan, Z.X., Koh, J.M., Koonin, E.V., Cheong, K.H.:Predator Dormancy is a Stable Adaptive Strategy due to Parrondo's Paradox. Adv. Sci. 7(3), 1901559 (2019)

42. Koh, J.M., Cheong, K.H.:Emergent preeminence of selfishness: an anomalous Parrondo perspective. Nonlinear Dyn. 98, 943-951 (2019)

43. Cheong, K.H., Wen, T., Lai, J.W.:Relieving Cost of Epidemic by Parrondo's Paradox: A COVID-19 Case Study. Adv. Sci. 7(24), $2002324(2020)$

44. Lai, J.W., Cheong, K.H.:Parrondo's paradox from classical to quantum: A review. Nonlinear Dyn. 100, 849-861 (2020)

45. Traulsen, A., Nowak, M.A., Pacheco, J.M.:Stochastic payoff evaluation increases the temperature of selection. J. Theory Biol. 244(2), 349-356 (2007)

46. Chen, X.J., Fu, F., Wang, L.:Interaction stochasticity supports cooperation in spatial prisoner's dilemma. Phys. Rev. E 78(5), 051120 (2008)

47. Li, J.Q., Park, J.H., Zhang, J.L., Chen, Z.Q., Dehmer, M.:The networked cooperative dynamics of adjusting signal strength based on information quantity. Nonlinear Dyn. 100(1), 831-847 (2020)

48. Szabó, G., Töke, C.:Evolutionary prisoner's dilemma game on a square lattice. Phys. Rev. E, 58(1), 69-73 (1998)

49. Tanimoto, J., Sagara, H.:Relationship between dilemma occurrence and the existence of a weakly dominant strategy in a twoplayer symmetric game, BioSystems 90(1), 105-114 (2007)
50. Wang, Z., Kokubo, S., Jusup, M., Tanimoto, J.:Dilemma strength as a framework for advancing evolutionary game theory: Reply to comments on "Universal scaling for the dilemma strength in evolutionary games". Phys. Life. Rev. 14, 56-58 (2015)

51. Xu, X.L., Chen, Z.Q., Si, G.Y. et al: The chaotic dynamics of the social behavior selection networks in crowd simulation. Nonlinear Dyn. 64(1), 117-126 (2011)

52. Xia, C.Y., Wang, L., Sun, S., Wang, J.:An SIR model with infection delay and propagation vector in complex networks. Nonlinear Dyn. 69(3), 927-934 (2012) 